\title{
Direction Reconstruction for the Radio Neutrino Observatory Greenland (RNO-G)
}

\section{Ilse Plaisier ${ }^{a, b}, *$ on behalf of the RNO-G Collaboration}

(a complete list of authors can be found at the end of the proceedings)

${ }^{a}$ DESY, Platanenallee 6, 15738 Zeuthen, Germany

${ }^{b}$ ECAP, Friedrich-Alexander-University Erlangen-Nuremberg, 91058 Erlangen, Germany

E-mail: ilse.plaisier@desy.de

\begin{abstract}
The Radio Neutrino Observatory Greenland (RNO-G) is planned to be the first large-scale implementation of the in-ice radio detection technique. It targets astrophysical as well as cosmogenic neutrinos with energies above $10 \mathrm{PeV}$. The deep component of a single RNO-G station consists of three strings with antennas to capture horizontal as well as vertical polarization. This contribution shows a model-based approach to reconstruct the arrival direction of the neutrinos with an RNO-G station. The timing of the waveforms is used to reconstruct the vertex position. The shape and amplitude of the waveform are used to reconstruct the viewing angle. Together with the signal polarization it will add up to the neutrino arrival direction. We present the method used and the achieved angular resolution using the deep component of an RNO-G station.
\end{abstract}

$37^{\text {th }}$ International Cosmic Ray Conference (ICRC 2021)

July 12th-23rd, 2021

Online - Berlin, Germany

\footnotetext{
*Presenter
} 

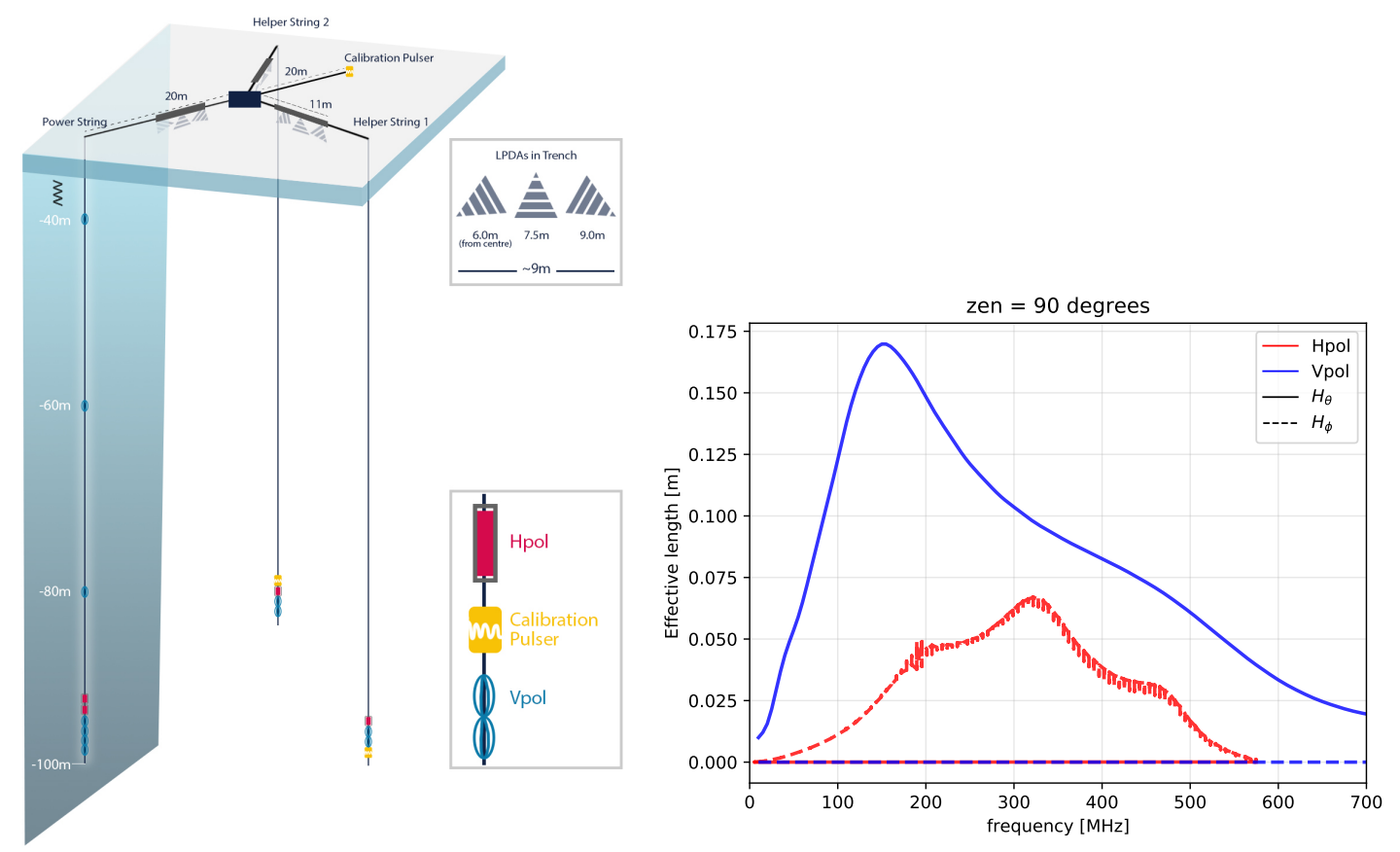

Figure 1: Left: A single RNO-G station consists of three strings of antennas (Hpol and Vpol) plus surface antennas (LPDAs), as well as three calibration pulsers located both deep in the ice and also at the surface. The string containing the phased array trigger is designated as the power string, while the two additional strings are designated as support strings. Right: The vector effective length of the RNO-G Vpol and Hpol. The Vpol is more broadband and has a higher gain than the Hpol, which is limited by the borehole geometry.

\section{Astrophysical neutrinos above $10 \mathrm{PeV}$}

Astrophysical neutrinos have been detected up to $10 \mathrm{PeV}$ energies [1] and promising correlations of neutrino arrival directions with Blazars [2] and Tidal Disruption Events [3] have been reported. For higher energies, the steeply falling flux requires large effective area neutrino detectors with fiducial volumes on the cubic kilometer scale. Radio neutrino detectors can deliver such large effective volumes by detecting the signals from particle showers in ice. The Radio Neutrino Observatory Greenland (RNO-G) will be the first large-scale neutrino radio detection experiment. To contribute to multi-messenger astronomy at higher energies and to find potential source correlations an accurate arrival direction reconstruction is needed. This will be discussed in these proceedings.

\section{Radio Neutrino Observatory Greenland (RNO-G)}

The start of the deployment of RNO-G is in June 2021 at Summit Station, Greenland. In the coming years, a total of 35 stations will be deployed with a spacing between stations of $1.5 \mathrm{~km}$. The station spacing is optimized such that most events will be detected by only a single station. Therefore, reconstructions and station design are optimized for inferring neutrino properties by just using a single station. A single RNO-G station as shown in Figure 1 consists of a deep component and a shallow component. The shallow component is reached less likely by a radio signal from 


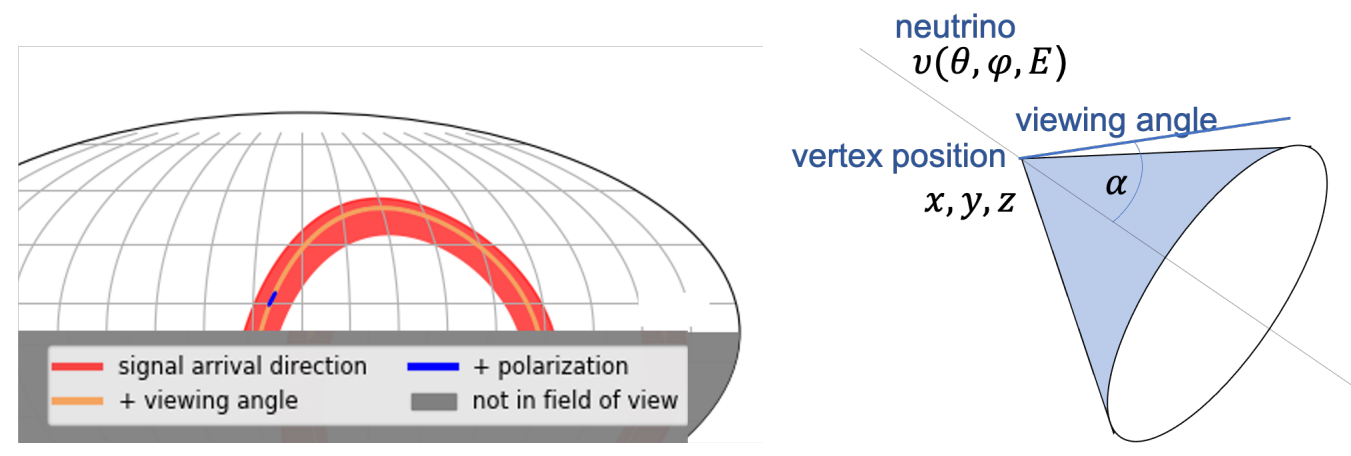

Figure 2: Left: Schematic of regions on-sky obtained by restricting the direction of the neutrino due to signal direction, viewing angle and polarization. A measure for the polarization is needed to limit the direction of the neutrino from a full cone to a small region. Right: Schematic of a neutrino interaction. The interaction point is referred to as the vertex position. The viewing angle, which is the angle between the shower axis and the radio signal propagating to a RNO-G station, takes values $\pm 7^{\circ}$ around the Cherenkov angle $\left(56^{\circ}\right)$.

within the ice. Therefore the reconstruction in this work is focussed on the deep component. Also, the performance of LPDA based stations has been shown previously by the ARIANNA experiment [4] and is applicable to RNO-G stations.

The deep component consists of three strings with a horizontal spacing of $20 \mathrm{~m}$ from the mid-point with a maximum depth of $100 \mathrm{~m}$. The horizontal spacing of the strings is optimized such that all three the strings need to see high enough SNR (signal-to-noise ratio) in order to get an azimuth reconstruction. The efficiency for each string to record a signal of SNR $>2.5$ is shown in Figure 7, right. One string is referred to as the power string and contains the trigger and is mainly used for vertex reconstruction. There are 7 antennas for vertical polarized electric-field (Vpols) and 2 for horizontal (Hpols). The other two strings have 2 Vpols, $1 \mathrm{Hpol}$ and 1 calibration pulser. These antennas are mainly for azimuthal reconstruction.

The antennas were optimized such that the geometry matches with the size of the borehole, which is 5.7 inches in radius. For the Hpol, a vertically oriented quadslot antenna design has been chosen. Figure 1 shows the antenna response of the Vpol and Hpol for the horizontal in terms of vector effective length $H_{\text {eff. }}$. The $H_{\text {eff }}$ is the immediate conversion factor between the electric-field and the voltage response in the antennas [5]. Clearly visible is the lower gain and smaller frequency bandwidth of the Hpol with respect to the Vpol. A contribution in the Vpol as well as Hpol is needed for a full polarization reconstruction.

\section{Direction Reconstruction}

Loosely, there are three ingredients needed to reconstruct the direction of the incoming neutrino: the radio signal direction, the polarization and the viewing angle. The viewing angle is the angle between the radio signal and the shower axis, as shown in Figure 2. The radio signal adds coherently on the Cherenkov angle (56 in ice). Higher frequencies fall off for viewing angles a few degrees off of the Cherenkov angle, and the signal becomes less pronounced. Therefore, the slope of the frequency spectrum can be used to determine the viewing angle. 


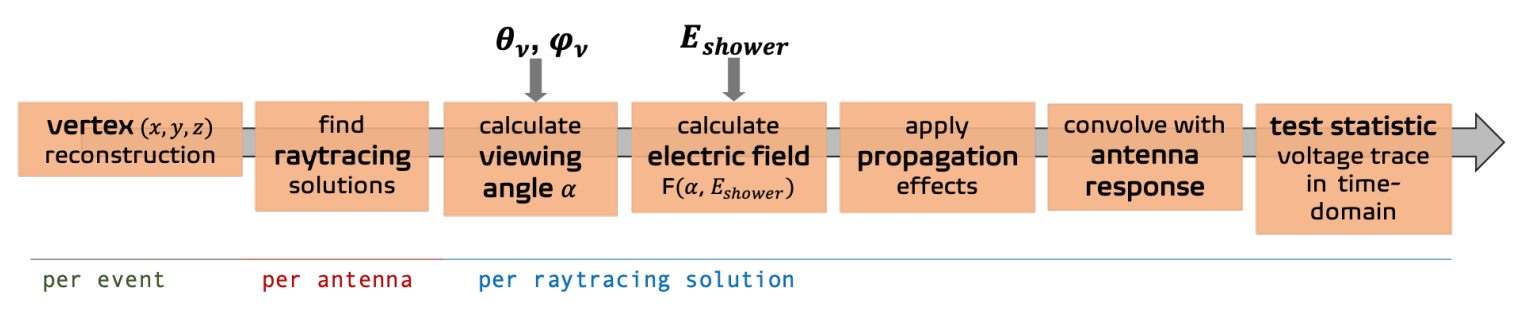

Figure 3: Schematic of steps taken in the fit.

Depending on the event, signals can typically still be observed with a viewing angle of $\pm 7^{\circ}$ around the Cherenkov angle. The signal direction can be obtained using the time-differences of the pulses in different channels. The Askaryan effect results in a polarization pointing towards the shower-axis, and therefore a contribution in the Vpol as well as Hpol will pinpoint the neutrino back to its origin. This is illustrated in Figure 2 left.

The neutrino simulations are performed with NuRadioMC $[5,6]$. In the simulations, the vertex position (position of neutrino interaction) is randomly generated. From this position, the propagation path of the radio signal is calculated using the the refractive index of the ice to each individual antenna. The density profile of the ice is not constant, and therefore besides the direct raypath (if the depth is monotonically decreasing or increasing along the path between emitter and receiver) a refracted raypath (if the path shows a turning point) can also be a solution. Due to the ice-air interface, the radio signal can be reflected and cause a reflected solution.

For a specific neutrino direction, the viewing angles at each antenna can be calculated using the raypaths. For the analysis presented in these proceeding, an analytic raytracing-solver is used, which calculates the raypath for a given start- and end-point only allowing to solve for exponential ice models. Other methods are currently being implemented in NuRadioMC, to support numerical raytracing [7] and a parabolic equations solver [8] to calculate the electric-field immediate at the antenna without the simplification of assuming ray optics.

Forward Folding To reconstruct the direction of the neutrino, an analytic model of the electric field-pulse is fitted directly to the measured voltages in the time-domain. We refer to this method as the forward folding technique. The performance of this method on cosmic-ray simulations is shown in [5], but has been significantly adapted for the neutrino case. A $\chi^{2}$ test statistic is used which is minimized for the neutrino azimuth angle, zenith angle and energy. In this approach, a model of the electric-field as emitted by the particle shower is used, the propagation effects and detector responses are then applied to calculate the expected voltage traces. Consequently, the $\chi^{2}$ is sample-wise minimized in the time-domain. This is schematically shown in Figure 3.

Fitting Procedure As a first step of the reconstruction, the vertex position is determined by calculating the timing differences between channel pairs and comparing these timing-differences with the expected timing-difference using ray-tracing. A 3D grid-scan is performed along a narrow band in the ice, where the region is determined by fitting a plane wave to the bottom channels to obtain the signal arrival direction. Second, the ray-tracing is performed from the reconstructed vertex to determine the travel paths and travel times for each ray-tracing solution. For each neutrino arrival direction, the viewing angles can be calculated using the ray paths of each raytracing 


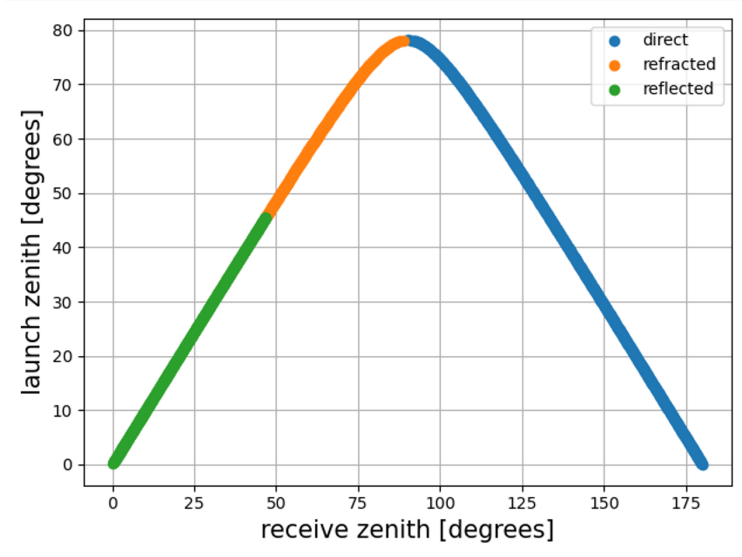

Figure 4: The launch zenith angle as function of received zenith angle. The signal arrival direction at the antennas can be reversed to the signal launch vector at the vertex position under the assumption of the ray type. The figure shows the mapping for a depth of $-97 \mathrm{~m}$, which is the depth of the reference channel used in the fitting procedure.

solution. The analytic-model of the electric-field (parametrized dependent on viewing angle and shower energy) is calculated for each pulse at each antenna. Each calculated electric-field trace is corrected for the propagation effects (such as attenuation) and convolved with the antenna and amplifier response to calculate voltage traces. Using the reconstructed signal direction via the plane wave fit, the ray type of can be determined of the triggering pulse as shown in Figure 4. The phased array channels are used as reference channels: by running a neutrino voltage-trace template through the traces, the position of the triggering pulse is determined, which sets the global timing. Also, the rough position of the other pulses in the other channels using the calculated travel times from the ray-tracing can be determined. The exact pulse positions are determined using correlation.

Simulation set The event set used to show the performance of the forward folding technique is a combination of an extension of the spectrum measured by IceCube and a GZK flux model [9]. For the simulations, the signal parameterization from [10] is used. The noise on the signal is dominated by thermal noise of the antennas itself. Currently, Rayleigh distributed noise is used. Once in-situ, more complete noise models can be obtained from measurements. In the simulation set, no charged current electron neutrinos are used. The current method depends strongly on an accurate model of the signal, which causes complications for electromagnetic showers, due to the LPM effect at high energies.

Results Figure 5 shows the resolution for the radio signal arrival direction, viewing angle and polarization. While signal zenith angle and viewing angle can be relatively accurately determined, this is not the case for the polarization. The polarization is the limiting factor for the neutrino arrival direction reconstruction. The signal arrival direction can be reconstructed at sub degree level, and the viewing angle at $\pm 0.5^{\circ}$.

Figure 6 shows the reconstructed space angle as function of energy and source zenith ( $90 \mathrm{deg}$ is horizontal). For events with a large signal contribution in the Hpol, a $\sigma_{68 \%}$ of $1.7^{\circ}$ is obtained. For small source zenith angles, due to geometry only the top of the cone is visible, and therefore 

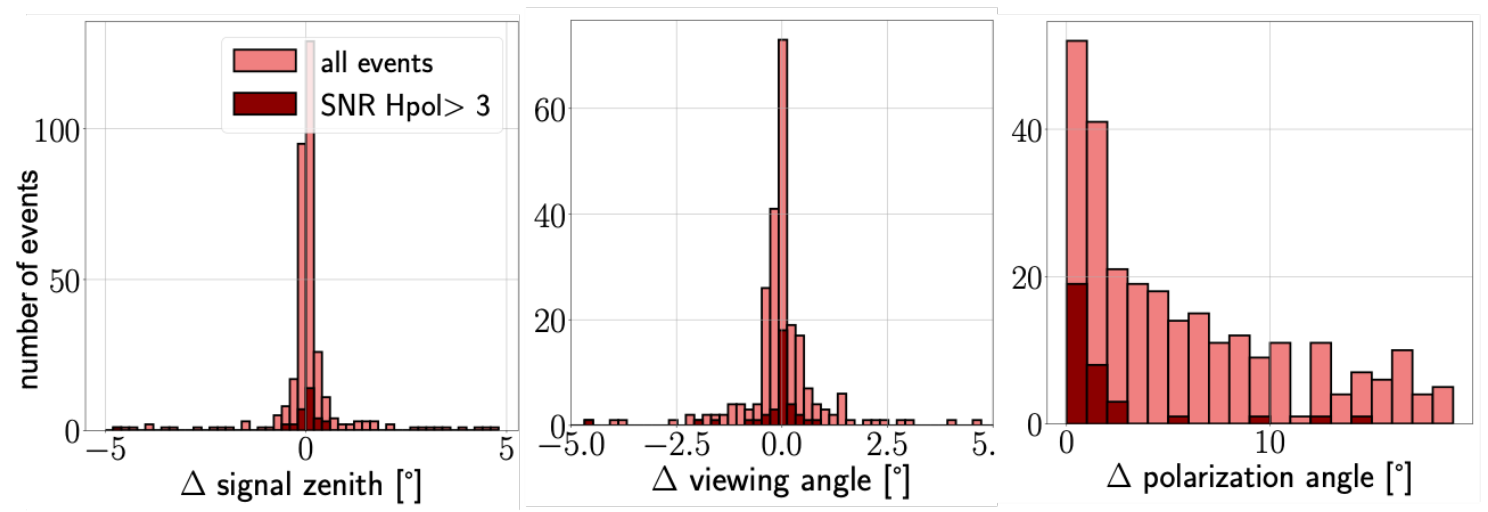

Figure 5: Reconstruction of the event parameters of the signal set for signal direction (left), middle (viewing angle) and polarization (right).
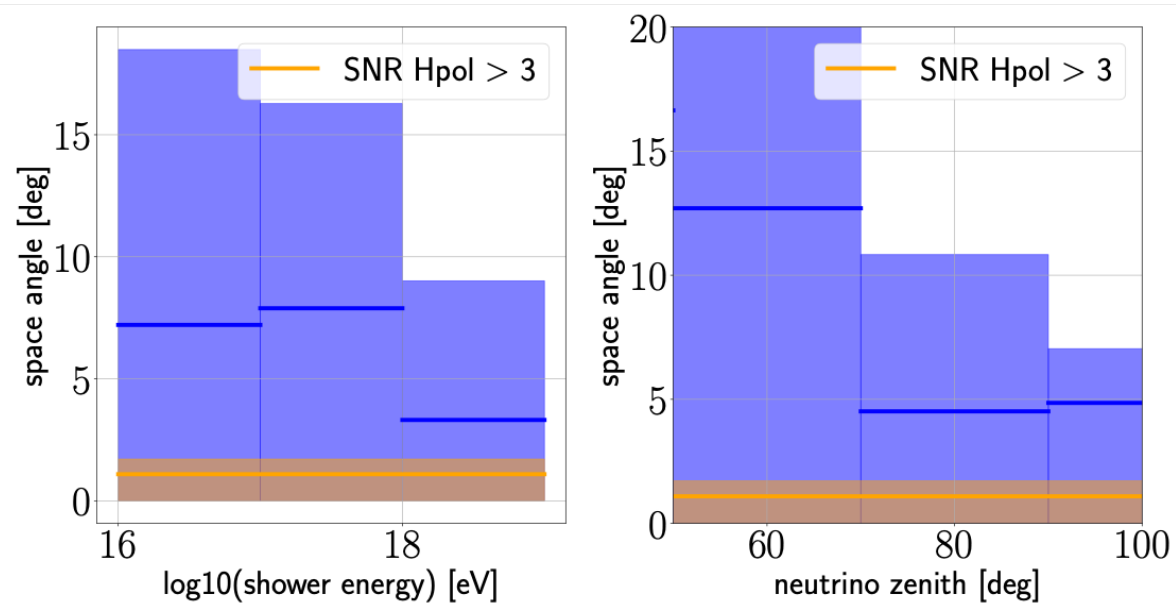

Figure 6: Obtainable space angle resolution as function of energy (left) and zenith (right) bins. Results for all events (blue) and a subsection of events with detectable Hpol contribution (orange, defined as signalto-noise ratio of 3) are shown. The thick lines correspond to $\sigma_{68 \%}$ and the shaded areas to $\sigma_{95 \%}$. The resolution improves with increasing energy and increasing source zenith $\left(90^{\circ}\right.$ horizontal). The events with Hpol contribution (orange) are grouped in one bin only.

the electric field contains no horizontal polarization. Therefore, the fraction of Hpol contribution events goes up for higher zeniths, resulting in a better angular resolution. For higher energies, Figure 7 left, shows an increasing fraction of events with SNR $>2.5$ for each zenith bin. Because of the large attenuation length in Greenland $(\mathrm{O}(1 \mathrm{~km}))$, compared to the thickness of the ice-sheet, most events at high energies will have amplitudes significantly higher than the trigger threshold. Therefore, the resolution for higher energies also improves.

\section{Implications for RNO-G science case}

RNO-G is sensitive to neutrinos from PeV energies and is therefore exploring a new parameter space. RNO-G has the potential to resolve the high-energy neutrino sky, identify sources or set 

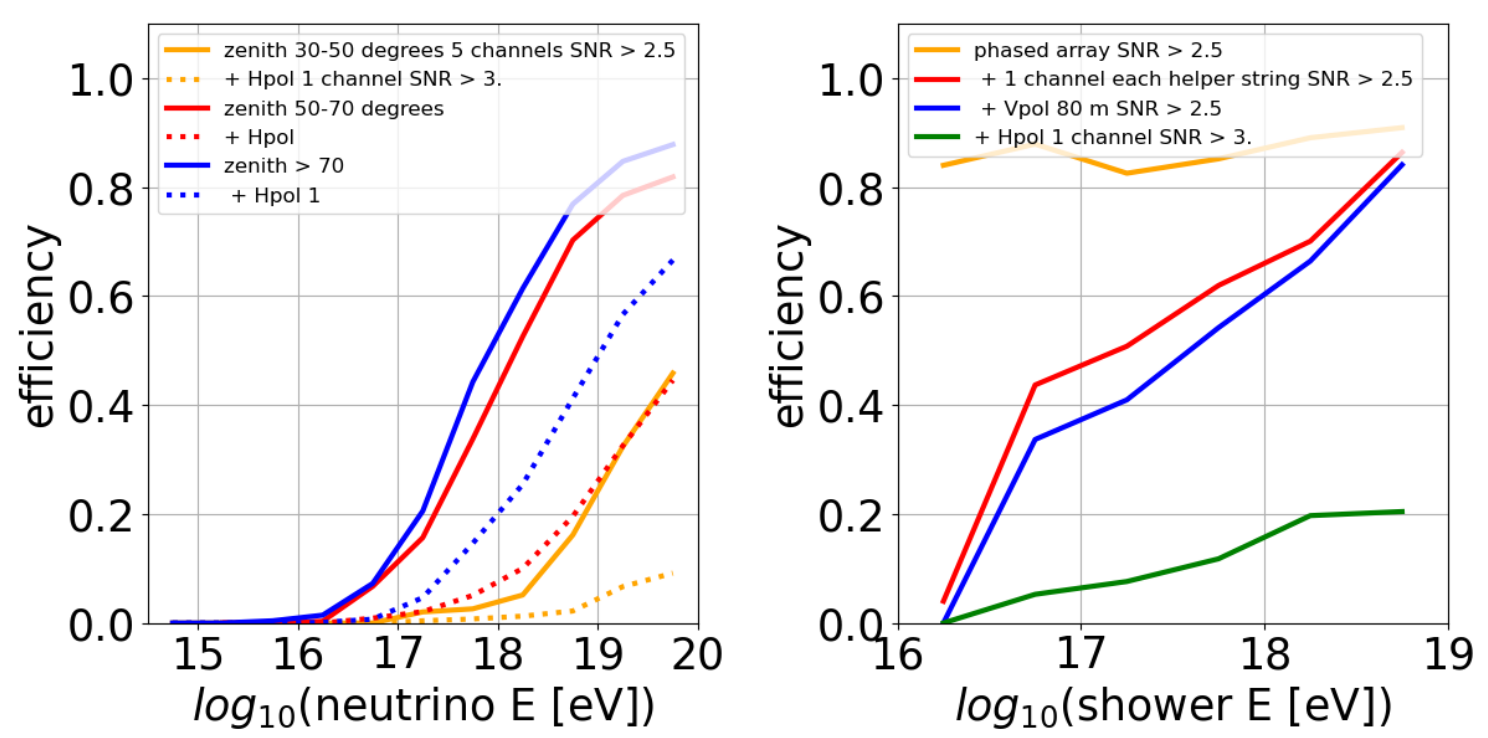

Figure 7: Left: Analysis efficiency for RNO-G versus energy and for different zenith bins. Right: Analysis efficiency for RNO-G versus energy. Signal in each string is needed in order to obtain azimuthal reconstruction (red) Addition of Hpol contribution is needed for neutrino direction reconstruction (green).

limits on the high-energy flux. RNO-G covers most of the northern hemisphere and is sensitive to among others the TA hotspot [11] and TXS 0506+056 [2]. Furthermore, RNO-G can exclude or verify the most optimistic cases for cosmogenic neutrino as shown in Figure 24 of [12]. More work needs to be done for a full study of the systematic uncertainties, besides only the noise fluctuations accounted for in this work. With the results shown here, an angular resolution of $\sigma_{68 \%}=1.7^{\circ}$ for events with $\mathrm{Hpol}$ contribution and a $\sigma_{68 \%}=8.5^{\circ}$ for all events, RNO-G will be suitable for the discovery of transients. The sensitivity of RNO-G to transients in shown in Figure 28 of [12]. When no timing constraint can be made, RNO-G will be dependent on multi-messenger approaches. Since the significance goes with $1 /\left(\right.$ space angle $^{2}$ ) that a multi-messenger observation belongs to the neutrino, having a large Hpol contribution is very important.

Regarding fundamental physics, mainly cross-section studies depend on angular resolution. Since the deep station is less sensitive to near horizontal events, a significant part of the sensitivity for cross-section measurements will also come from the surface component, which is not studied in this work.

\section{References}

[1] ICECube collaboration, Evidence for High-Energy Extraterrestrial Neutrinos at the IceCube Detector, Science 342 (2013) 1242856-1242856.

[2] M. Aartsen et al., Neutrino emission from the direction of the blazar TXS 0506+056 prior to the IceCube-170922A alert, Science 361 (2018) 147-151.

[3] R. Stein et al., A tidal disruption event coincident with a high-energy neutrino, Nature Astronomy 5 (2021) 510-518. 
[4] ARIANNA collaboration, Probing the angular and polarization reconstruction of the ARIANNA detector at the South Pole, JINST 15 (2020) P09039 [2006. 03027].

[5] C. Glaser, A. Nelles, I. Plaisier, C. Welling, S. W. Barwick, D. García-Fernández et al., Nuradioreco: a reconstruction framework for radio neutrino detectors, The European Physical Journal C 79 (2019) .

[6] C. Glaser, D. García-Fernández, A. Nelles, J. Alvarez-Muñiz, S. W. Barwick, D. Z. Besson et al., Nuradiomc: simulating the radio emission of neutrinos from interaction to detector, The European Physical Journal C 80 (2020) .

[7] T. Winchen, RadioPropa - A Modular Raytracer for In-Matter Radio Propagation, EPJ Web of Conferences 216 (2019) 03002.

[8] S. Prohira et al., Modeling in-ice radio propagation with parabolic equation methods, Physical Review D 103 (2021) .

[9] A. van Vliet, R. A. Batista and J. R. Hörandel, Determining the fraction of cosmic-ray protons at ultrahigh energies with cosmogenic neutrinos, Physical Review D 100 (2019).

[10] J. Alvarez-Muñiz, W. R. Carvalho, M. Tueros and E. Zas, Coherent Cherenkov radio pulses from hadronic showers up to EeV energies, Astroparticle Physics 35 (2012) 287-299.

[11] R. U. Abbasi et al., Indications of intermediate-scale anisotropy of cosmic rays with energy greater than $57 \mathrm{EeV}$ in the Northern sky measured with the surface detector of the Telescope Array Experiment, The Astrophysical Journal 790 (2014) L21.

[12] RNO-G collaboration, Design and sensitivity of the Radio Neutrino Observatory in Greenland (RNO-G), Journal of Instrumentation 16 (2021) P03025. 


\section{Full Authors List: RNO-G Collaboration}

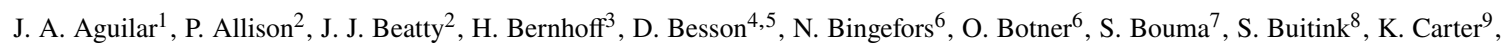
M. Cataldo ${ }^{7}$, B. A. Clark ${ }^{10}$, Z. Curtis-Ginsberg ${ }^{11}$, A. Connolly ${ }^{2}$, P. Dasgupta ${ }^{1}$, S. de Kocker ${ }^{12}$, K. D. de Vries ${ }^{12}$, C. Deaconu ${ }^{11}$, M. A. DuVernois ${ }^{13}$, C. Glaser ${ }^{6}$, A. Hallgren ${ }^{6}$, S. Hallmann ${ }^{14}$, J. C. Hanson ${ }^{15}$, B. Hendricks ${ }^{16}$, B. Hokanson-Fasig ${ }^{13}$, C. Hornhuber ${ }^{4}$, K. Hughes ${ }^{11}$, A. Karle ${ }^{13}$, J. L. Kelley ${ }^{13}$, S. R. Klein ${ }^{17}$, R. $\operatorname{Krebs}^{16}$, R. Lahmann ${ }^{7}$, U. Latif ${ }^{12}$, M. Magnuson ${ }^{4}$, T. Meures ${ }^{13}$, Z. S. Meyers ${ }^{14,7}$, K. Mulrey ${ }^{8}$, A. Nelles ${ }^{14,7}$, A. Novikov ${ }^{4}$, E. Oberla ${ }^{11}$, B. Oeyen ${ }^{18}$, H. Pandya ${ }^{8}$, I. Plaisier ${ }^{7,14}$, L. Pyras ${ }^{14,7}$, D. Ryckbosch ${ }^{18}$, O. Scholten ${ }^{12,19}$, D. Seckel ${ }^{20}$, D. Smith ${ }^{11}$, D. Southall ${ }^{11}$, J. Torres ${ }^{2}$, S. Toscano ${ }^{1}$, D. Tosi ${ }^{13}$, D. J. Van Den Broeck ${ }^{12,8}$, N. van Eijndhoven ${ }^{12}$, A. G. Vieregg ${ }^{12}$, C. Welling ${ }^{7,14}$, S. Wissel ${ }^{16,9}$, R. Young ${ }^{4}$, and A. Zink ${ }^{7}$

${ }^{1}$ Université Libre de Bruxelles, Science Faculty CP230, B-1050 Brussels, Belgium

${ }^{2}$ Dept. of Physics, Center for Cosmology and AstroParticle Physics, Ohio State University, Columbus, OH 43210, USA

${ }^{3}$ Uppsala University, Dept. of Engineering Sciences, Division of Electricity, Uppsala, SE-752 37, Sweden

${ }^{4}$ University of Kansas, Dept. of Physics and Astronomy, Lawrence, KS 66045, USA

${ }^{5}$ National Nuclear Research University MEPhI, Kashirskoe Shosse 31, 115409, Moscow, Russia

${ }^{6}$ Uppsala University, Dept. of Physics and Astronomy, Uppsala, SE-752 37, Sweden

${ }^{7}$ Erlangen Center for Astroparticle Physics (ECAP), Friedrich-Alexander-University Erlangen-Nuremberg, 91058 Erlangen, Germany

${ }^{8}$ Vrije Universiteit Brussel, Astrophysical Institute, Pleinlaan 2, 1050 Brussels, Belgium

${ }^{9}$ Physics Dept. California Polytechnic State University, San Luis Obispo CA 93407, USA

${ }^{10}$ Dept. of Physics and Astronomy, Michigan State University, East Lansing MI 48824, USA

${ }^{11}$ Dept. of Physics, Enrico Fermi Inst., Kavli Inst. for Cosmological Physics, University of Chicago, Chicago, IL 60637, USA

${ }^{12}$ Vrije Universiteit Brussel, Dienst ELEM, B-1050 Brussels, Belgium

${ }^{13}$ Wisconsin IceCube Particle Astrophysics Center (WIPAC) and Dept. of Physics, University of Wisconsin-Madison, Madison, WI 53703, USA

${ }^{14}$ DESY, Platanenallee 6, 15738 Zeuthen, Germany

${ }^{15}$ Whittier College, Whittier, CA 90602, USA

${ }^{16}$ Dept. of Physics, Dept. of Astronomy \& Astrophysics, Penn State University, University Park, PA 16801, USA

${ }^{17}$ Lawrence Berkeley National Laboratory, Berkeley, CA 94720, USA

${ }^{18}$ Ghent University, Dept. of Physics and Astronomy, B-9000 Gent, Belgium

${ }^{19}$ Kapteijn Institute, University of Groningen, Groningen, The Netherlands

${ }^{20}$ Dept. of Physics and Astronomy, University of Delaware, Newark, DE 19716, USA 